\title{
AN UPPER BOUND TO THE SPACE DENSITY OF INTERSTELLAR COMETS
}

\author{
M. Jura \\ Department of Physics and Astronomy, University of California, Los Angeles CA 90095
}

\begin{abstract}
Two well-studied white dwarfs with helium-dominated atmospheres (DBs) each possess less hydrogen than carried by a single average-mass comet. Plausibly, the wind rates from these stars are low enough that most accreted hydrogen remains with the star. If so, and presuming their nominal effective temperatures, then these DBs have been minimally impacted by interstellar comets during their $50 \mathrm{Myr}$ cooling age; interstellar iceballs with radii between $10 \mathrm{~m}$ and $2 \mathrm{~km}$ contain less than $1 \%$ of all interstellar oxygen. This analysis suggests that most stars do not produce comets at the rate predicted by "optimistic" scenarios for the formation of the Oort cloud.
\end{abstract}

Subject headings: interstellar medium - planetary systems - white dwarfs

\section{INTRODUCTION}

There are two reasons to imagine that interstellar comets could be widespread. First, they could be an important as-yet undetected reservoir of interstellar oxygen. Second, models for the formation of the solar system's Oort cloud predict that more comets are ejected into the interstellar medium than remain gravitationally bound to the Sun. If our solar system is at all typical, then the birth of stars and planets might commonly lead to the creation of many interstellar comets.

Despite decades of effort, analysis of the rate of appearance of new comets in the solar system has not provided a strong constraint on the space density of interstellar comets. Substantial progress may require another approach. In this spirit, Shull \& Stern (1995) argued that one possible explanation for the "low" observed frequency of gamma ray bursts arising from the Milky Way is that there are few interstellar comets impacting upon neutron stars. However, there are enough uncertainties in this approach, that their result that fewer than $4 \%$ of stars have Oort clouds similar to the Sun's is not completely firm. While also employing degenerate stars as a tool, here, we proceed somewhat differently. We argue that the small amounts of hydrogen accumulated by warm DB white dwarfs significantly limits the rate of iceball impact with these stars and therefore the space density of interstellar comets. Our method requires combining an understanding of the interstellar medium with the physics of external pollution of white dwarfs. We therefore subdivide this Introduction into subsections to describe the different threads of our approach. 


\subsection{Interstellar Oxygen}

Including both atoms in the gas phase and those estimated to be contained within dust grains, the abundance of oxygen within diffuse clouds, $n(\mathrm{O}) / n(\mathrm{H})$, is within $15 \%$ of $5.1 \times 10^{-4}$ (Cartledge et al. 2004). While this value is in agreement with recent determinations of the solar photospheric oxygen abundance of $n(\mathrm{O}) / n(\mathrm{H})=4.6 \times 10^{-4}$ (Asplund et al. 2004) or $n(\mathrm{O}) / n(\mathrm{H})$ $=5.8 \times 10^{-4}$ (Caffau et al. 2008), the true solar abundance may be as high as $n(\mathrm{O}) / n(\mathrm{H})=7.2$ $\times 10^{-4}$ (Delahave \& Pinsonneault 2006). Even with the problematical assumption that the interstellar and solar oxygen abundances are the same, the interstellar oxygen abundance is $\sim 50 \%$ uncertain. The possibility of a missing reservoir of interstellar oxygen has been considered for decades (Greenberg 1974), and here we re-assess the hypothesis that appreciable oxygen is carried within interstellar comets. Furthermore, although not directly related to the question of the total abundance of interstellar oxygen, the observed decrease of $n(\mathrm{O}) / n(\mathrm{H})$ with column density (Cartledge et al. 2004; Jenkins 2009) is not well understood and presents a "crisis" in our understanding of the behavior and distribution of interstellar oxygen (Whittet 2010).

With an interstellar density of hydrogen nuclei near the Sun of $1.15 \mathrm{~cm}^{-3}$ (Bohlin et al. 1978 ) and with $n(\mathrm{O}) / n(\mathrm{H})=5.1 \times 10^{-4}$, the mass density of interstellar oxygen is $1.6 \times 10^{-26} \mathrm{~g} \mathrm{~cm}^{-3}$. Next, we compare this estimate with observational bounds and theoretical expectations for the space density interstellar comets and the amount of oxygen that they contain.

\subsection{Interstellar Comets?}

To date, all observed solar system comets appear to have always been gravitationally bound to the Sun. Consequently Whipple (1975) and Sekanina (1976) placed upper limits of $2 \times 10^{-26}$ $\mathrm{g} \mathrm{cm}^{-3}$ and $4 \times 10^{-26} \mathrm{~g} \mathrm{~cm}^{-3}$, respectively, to the mass density of interstellar comets. Using the same approach but with updated parameters, Francis (2005) placed an upper bound to the space density of interstellar comets of $4.5 \times 10^{-4} \mathrm{AU}^{-3}$. With an average mass per comet as much as $1.2 \times 10^{18} \mathrm{~g}$ (Francis 2005), and assuming about 50\% of a comet's mass is oxygen (Greenberg 1998), then the upper bound to the mass density of oxygen within interstellar comets is $8 \times 10^{-26}$ $\mathrm{g} \mathrm{cm}^{-3}$ - larger than the amount of gas-phase interstellar oxygen (Stern \& Shull 1990).

Conventional dynamic models for the formation of the Oort cloud from objects in the inner solar system (Duncan et al. 1987) predict that there could be as many as 50 comets ejected into the interstellar medium for every comet that remains bound to the Sun. Unconventional models for the Oort cloud (Levison et al. 2010) also must require significant numbers of interstellar comets. An "optimistic" model for the space density of interstellar comets presumes that all stars form massive comet clouds similar to the solar system's Oort cloud and that most of these comets are lost into the interstellar medium. With this picture, Stern (1990) predicted that the space density of interstellar comets could be as high as $2 \times 10^{-43} \mathrm{~cm}^{-3}$ (or $7 \times 10^{-4} \mathrm{AU}^{-3}$ ), comparable to the observational upper limit of Francis (2005). Consequently, "optimistic" models predict that 
occasional interstellar comets can be observed within the solar system (McGlynn \& Chapman 1989; Sen \& Rama 1993). However, since the mass of the Oort cloud, the fraction of comets that are ejected into the interstellar medium and the fraction of stars that create analogs to the Oort cloud are each uncertain by a factor of $\sim 10$ (Stern 1990; Shull \& Stern 1995; Weissman 1996; Dones et al. 2004), the "optimistic" scenario may vastly overestimate the true space density of interstellar comets.

\subsection{A New Approach}

The basic idea of this paper is to use white dwarfs with helium dominated atmospheres (DBs) to limit the space density of interstellar comets. The large majority of white dwarfs have atmospheres which are hydrogen dominated and these stars are classified as DAs (Liebert et al. 1986; Sion et al. 1988). The standard scenario is that gravitational settling of heavy elements is very effective, and therefore, with sufficient time, essentially all of the hydrogen is located in the star's outer envelope and dominates the composition of the photosphere (Hansen \& Liebert 2003). A minority of white dwarfs have so little hydrogen that they have helium dominated atmospheres even though all their hydrogen resides in the star's outer envelope. If lines from neutral helium are detected, they are classified as DBs. If too cool for neutral helium to be detectable yet they display heavy elements in their spectra, they are classified as DZs.

Two recent surveys show that white dwarfs accumulate hydrogen from an external source because there is a a correlation between the average hydrogen mass and a DB's or DZ's cooling age (Dufour et al. 2007; Voss et al. 2007). The typical rate accretion rate is near $6 \times 10^{5} \mathrm{~g} \mathrm{~s}^{-1}$ (Jura \& Xu 2010), but there are large variations. In this paper, we focus on the $\sim 50 \%$ of DBs with only upper bounds to the mass of accumulated hydrogen (Voss et al. 2007).

The mass of the convective zone where the accreted material resides is a strong function of the star's effective temperature. Because DBs with $T_{*}<20,000 \mathrm{~K}$ have convective zones that are 10,000 times more massive than DBs with $T_{*}>22,000 \mathrm{~K}$ (Koester 2009), these two temperature classes may display external pollution quite differently.

There is relatively little hydrogen in DBs warmer than 22,000 K. A conservative upper bound from optical and ultraviolet observations of most "warm" DBs is that $n(\mathrm{H}) / n(\mathrm{He})<10^{-4}$ (Beauchamp et al. 1999; Voss et al. 2007). Since the mass of the outer convective zones of these warm DBs is at most $5 \times 10^{21} \mathrm{~g}$ (Koester 2009), these stars have accumulated less than $\sim 10^{17} \mathrm{~g}$ of hydrogen. As described in Section 3, two particularly well studied DBs, GD 190 and BPM 17088, have accumulated less than $4 \times 10^{15} \mathrm{~g}$ of hydrogen, roughly the amount of this element carried in a single average-size comet. At the "typical" accretion rate of $6 \times 10^{5} \mathrm{~g} \mathrm{~s}^{-1}$ experienced by the cool DBs, these two stars would accumulate the upper bound to their hydrogen in less than $10^{3} \mathrm{yr}$, much less time than their cooling ages of 50 Myr. Either the warm DBs do not accrete at the same rate as many cooler DBs, or they lose almost all their acquired hydrogen in a wind. 
In Section 2 we elaborate in detail upon the "naive" idea that warm DBs have not accumulated much hydrogen simply because they have not accreted from ice-rich asteroids, comets or gas from the interstellar medium - the current best candidates as the sources for hydrogen in cooler DBs. While this argument hardly seems remarkable, there are interesting consequences. Using the toy model for a DB's hydrogen budget developed in Section 2, in Section 3 we constrain the mass carried within interstellar comets. In Section 4, we outline observational possibilities while in Section 5 we present our conclusions.

\section{TOY MODEL FOR A WARM DB'S HYDROGEN BUDGET}

\subsection{Model Description}

We compute the amount of hydrogen in "warm" DBs for a regime when essentially all of its hydrogen of mass, $M(H)$ has risen to the surface layers and is well mixed in the outer convective zone of mass $M_{c v z}$. There may be a very thin non-convective photosphere lying above the convective zone, but we assume that the photosphere and the convective zone have the same composition (Dupuis et al. 1992; $\mathrm{Su} \& \mathrm{Li} 2010$ ). If hydrogen is accreted with rate $\dot{M}_{a c c}(H)$ and the total mass loss rate is $\dot{M}_{\text {wind }}$, then:

$$
\dot{M}(H)=\dot{M}_{a c c}(H)-\frac{M(H)}{M_{c v z}} \dot{M}_{w i n d}
$$

Equation (1) is mathematically equivalent to the expression governing the abundance of heavy atoms in the outer layer of a white dwarf where the atoms are "lost" because of downward settling (Koester 2009) instead of our case where hydrogen atoms are lost in an outward flow in a wind.

For our purposes here, there is a crucial difference between accretion of gas from the interstellar medium and accretion from large solid objects such as asteroids. There is no reason to think that a quasi-spherical stellar wind strongly inhibits the accretion from a flat disk produced by tidal disruption of a parent body (Jura 2003). In contrast, such a wind can physically block interstellar gas accretion. In particular, while winds of $10^{6} \mathrm{~g} \mathrm{~s}^{-1}$ can prevent interstellar accretion at the typical rate, once accreted hydrogen is diluted into a helium-dominated envelope, much higher wind outfows are required to inhibit the build-up of observable amounts of hydrogen.

It is useful to introduce the characteristic time for the wind to deplete the mass in the convective zone, $t_{d e p l}$ :

$$
t_{d e p l}=\frac{M_{c v z}}{\dot{M}_{\text {wind }}}
$$

If $M_{c v z}, \dot{M}_{w i n d}$ and $\dot{M}_{a c c}$ are independent of time, the solution to Equation (1) is:

$$
M(H)[t]=M(H)[0] e^{-t / t_{d e p l}}+\dot{M}_{a c c}(H) t_{d e p l}\left(1-e^{-t / t_{d e p l}}\right)
$$

Although Equation (3) provides a guide to our results, it is inexact because $M_{c v z}$ is not constant during the star's cooling. In the numerical solutions to Equation (1) presented below, we fit 
the detailed calculations for $M_{c v z}$ in a $0.6 \mathrm{M} \odot$ DB without hydrogen by Koester (2009) and Gautschy \& Althaus (2002) in the temperature interval 22,000 $\mathrm{K}<T_{*}<28,000 \mathrm{~K}$ with the expression:

$$
M_{c v z} \approx 1.5 \times 10^{21}\left(\frac{25,000}{T_{*}}\right)^{10}(\mathrm{~g})
$$

Also, we relate the star's effective temperature to its cooling age by fitting the calculations of Bergeron 11 for a $0.6 M_{\odot}$ DB white dwarf with:

$$
t \approx 49.7\left(\frac{22,000}{T_{*}}\right)^{5} \quad(\mathrm{Myr})
$$

Because $M_{c v z}$ increases as the star ages, then, as can be seen from Equation (1), other factors being equal, the loss rate of hydrogen is diminished. Consequently, instead of reaching an asymptotic value as predicted by Equation (3), as illustrated in Figures 1-3, $M(H)$ continues to increase with time.

\subsection{Boundary Condition}

We set $t=0$ for $T_{*}=28,000 \mathrm{~K}$, since at this moment in a DB's cooling, $M_{c v z}$ becomes large enough that Equation (1) can be sensibly employed. The value of $M(H)[0]$ is sensitive to the white dwarf's previous history (Unglaub \& Bues 2000). However, since the time for a $0.6 \mathrm{M}_{\odot} \mathrm{DB}$ to cool from $T_{*}=28,000 \mathrm{~K}$ to $T_{*}=22,000 \mathrm{~K}$ is about $35 \mathrm{Myr}$, then in most cases of interest, $t>t_{\text {depl }}$ since we focus on wind loss rates greater than $10^{6} \mathrm{~g} \mathrm{~s}^{-1}$. If so, then the exact value of $M(H)[0]$ is unimportant. In all cases, we adopt $M(H)[0]=1 \times 10^{15} \mathrm{~g}$, a conservative upper bound for the mass of hydrogen in EC 20058-5234 (Petitclerc et al. 2005), a well studied DB with $T_{*}=28,000$ K (Sullivan et al. 2008).

\subsection{White Dwarf Winds?}

By far, the largest uncertainty in Equation (1) is the stellar wind rate from white dwarfs. Unglaub (2008) has computed that radiatively-driven winds from white dwarfs with $T_{*}<30,000$ K do not occur. Quite possibly, therefore, we should adopt $\dot{M}_{\text {wind }}=0$. In contrast, in order to explain the presence of small amounts of dredged-up carbon in warm DBs, Fontaine \& Brassard (2005) have proposed that $\dot{M}_{\text {wind }}$ is as high as $\sim 10^{13} \mathrm{~g} \mathrm{~s}^{-1}$ for $T_{*}>20,000 \mathrm{~K}$. However, the physical explanation for this putative wind is not provided. One possibility might be that the white dwarfs have hot coronae which could drive a wind (Lamers \& Cassinelli 1999). In fact, white dwarfs do have convective envelopes and there could be some transfer of the convective energy flux into heating the outer layers of the gas.

\footnotetext{
${ }^{1}$ see http://www.astro.umontreal.ca/ bergeron/CoolingModels/Tables/Table_DB
} 
We use two lines of evidence to bound the wind rates from warm white dwarfs. First, these stars are not X-ray sources (O’Dwyer et al. 2003). Assume a wind outflow speed near the white dwarf's characteristic escape velocity, $V_{e s c}$, of $4000 \mathrm{~km} \mathrm{~s}^{-1}$ (Lamers \& Cassinelli 1999) and consider the kinetic energy flow carried by the wind, $1 / 2 \dot{M}_{\text {wind }} V_{\text {esc }}^{2}$. Much of this energy is deposited into the surrounding interstellar medium to create a hot bubble. Using the result that $L_{X}<4 \times 10^{27}$ $\operatorname{erg~s}^{-1}$ (Musielak et al. 2003) for GD 358, a DB with $T_{*}=27,000$ (Provencal et al. 2009), it seems that $\dot{M}_{\text {wind }}<\sim 5 \times 10^{10} \mathrm{~g} \mathrm{~s}^{-1}$. (This limit could be relaxed if the X-rays are deposited in a region greater than the 5".1 circle on the sky employed by Musielak et al. (2003) in their data analysis. However, as can be seen from their Figure 2, there is no extended X-ray emission.)

Second, some white dwarfs accrete and therefore are not losing a large amount of mass in a wind. Consider SDSS $1228+1040$ with $T_{*}=22,000 \mathrm{~K}$, the hottest white dwarf known to be heavily polluted and possess a dust disk (Gaensicke et al. 2006, Brinkworth et al. 2009, Melis et al. 2010). Because this DA does not have a convective envelope (Koester 2009), Equation (1) does not apply. Instead, to accumulate heavy elements, it is necessary that $\dot{M}_{\text {wind }}<\dot{M}_{a c c}$. From the formalism and parameters given by Koester (2009), the magnesium abundance reported by Gaensicke et al. (2007) and the presumption that $\sim 10 \%$ of the mass of the material is magnesium (see, for example, Klein et al. (2010)), then the heavy atom accretion rate is about $2 \times 10^{9} \mathrm{~g} \mathrm{~s}^{-1}$. This value is within the range of $3 \times 10^{8} \mathrm{~g} \mathrm{~s}^{-1}$ to $\sim 10^{10} \mathrm{~g} \mathrm{~s}^{-1}$ inferred for most white dwarfs with dust disks (Kilic et al. 2006; von Hippel et al. 2007; Farihi et al. 2009). It seems likely that the wind rate from this star is less than $10^{10} \mathrm{~g} \mathrm{~s}^{-1}$. (We assume that this DA without a convective envelope has the same mass loss rate as a DB with a low mass convective envelope. Therefore, this argument is not completely certain.)

The source of the hydrogen in DBs with $T_{*}<20,000 \mathrm{~K}$ Is uncertain. If some of these stars accrete interstellar gas with the typical time-averaged hydrogen accretion rate given by Voss et al. (2007) of $\sim 10^{6} \mathrm{~g} \mathrm{~s}^{-1}$, then $\dot{M}_{\text {wind }}$ likely is less than this value. Because warm DBs have much less

massive convective envelopes than cool DBs, there is no reason to imagine that $\dot{M}_{\text {wind }}$ increases dramatically with effective temperature. We conclude that the wind rates from warm DBs likely are less than $10^{10} \mathrm{~g} \mathrm{~s}^{-1}$ and plausibly less than $10^{6} \mathrm{~g} \mathrm{~s}^{-1}$.

Besides a wind, white dwarfs also may lose mass in explosive events as occurs in novae. Because there is no evidence that isolated white dwarfs undergo such events nor is there any theoretical reason to expect them, we ignore this possibility.

\subsection{Interstellar Gas Accretion?}

Although considered for many years, the physics of accreting interstellar hydrogen gas by white dwarfs is not well understood. It seems, for example, that accretion at the Bondi-Hoyle rate is excluded (Farihi et al. 2010). DBs younger than 50 Myr have performed less than one full oscillation through the Galactic Plane. Therefore, these stars may have only encountered hot, low 
density material and consequently have accreted interstellar gas at a negligible rate during their entire cooling age. Because accretion of interstellar gas-phase hydrogen can only strengthen the upper limits to asteroidal and cometary accretion discussed below, we neglect this possibility.

\subsection{Accretion of asteroids with ice?}

In our calculations for asteroidal accretion, we assume that $\dot{M}_{a c c}(H)=6 \times 10^{5} \mathrm{~g} \mathrm{~s}^{-1}$, a characteristic hydrogen accretion rate for DBs with $T_{*}<20,000 \mathrm{~K}$ although there is a large variation in this rate (Voss et al. 2007). We show in Figure 1 more detailed solutions to Equation (1). In these models, we see that if $\dot{M}_{\text {wind }}<10^{10} \mathrm{~g} \mathrm{~s}^{-1}$, then DBs typically accumulate more hydrogen than observed. This means that DBs warmer than 22,000 K either have "strong" winds or they do not accrete from ice-rich asteroids.

\subsection{Oort Cloud Analog Cometary Accretion?}

Alcock et al. (1986) proposed that direct impact by comets can produce observable signatures in a white dwarf's photosphere. Here, we estimate the hydrogen pollution of a DB white dwarf by comets in an analog to the solar system's Oort cloud. According to Francis (2005), 0.8 dynamically new comets $\mathrm{AU}^{-1} \mathrm{yr}^{-1}$ arrive in the inner solar system. With a flat distribution of perihelia, the expected rate of collisions onto a star of radius $0.013 \mathrm{R}_{\odot}$ is $4.8 \times 10^{-5} \mathrm{yr}^{-1}$. With an average comet mass somewhere between $5.6 \times 10^{16} \mathrm{~g}$ and $1.2 \times 10^{18} \mathrm{~g}$ (Francis 2005) and the assumption that 5\% of the comet's mass is hydrogen (Greenberg 1998), then the hydrogen accretion rate is expected to lie between $4 \times 10^{3} \mathrm{~g} \mathrm{~s}^{-1}$ and $9 \times 10^{4} \mathrm{~g} \mathrm{~s}^{-1}$. To be conservative, we adopt the lower bound and show results from Equation (1) in Figure 2. We see that if $\dot{M}_{\text {wind }}<10^{8} \mathrm{~g} \mathrm{~s}^{-1}$, then as long as $T_{*}>22,000 \mathrm{~K}$, the star accumulates more hydrogen than allowed for the typical upper bound for DBs obtained from optical spectra (Beauchamp et al. 1999). Therefore, if the winds from these white dwarfs are plausibly weak, the mass of their analogs to Oort cluds are smaller than the solar system's. Either these stars never had many primoridal comets or they lost their comets when the star evolved on the Asymptotic Giant Branch (Parrtiott \& Alcock 1998).

We assume that the comet's hydrogen is visible in the star's outer convective zone. In fact, during its high-speed impact, the iceball might penetrate beneath the convective zone. Following Alcock et al. (1986), we assume that the material vaporized from the comet has such a high entropy that it quickly rises to the surface of the star and therefore could be detected. 


\section{LIMITS ON INTERSTELLAR COMETS}

Having argued above that the well known sources of hydrogen accretion are not important in many warm DBs, we now proceed to estimate the space density of interstellar comets.

\subsection{Computed Accretion Rate}

We assume that the comets have hyperbolic orbits with speed relative to the white dwarf of $V_{w d}$. If the comet impacts a white dwarf of radius $R_{*}$ and mass $M_{*}$, then it is $100 \%$ accreted; we assume there is no accretion from a near-miss. We treat the ambient interstellar iceballs as a continuous fluid with a hydrogen mass density, $\rho_{b a l l}(H)$. Because the escape speed from the surface of the white dwarf is much greater than $V_{w d}$, then the total mass of accreted hydrogen, $M_{a c c}(H)$ is (see, for example, Spitzer (1978)):

$$
M_{a c c}(H) \approx 2 \pi G M_{*} R_{*} \int \frac{\rho_{\text {ball }}(H)[t]}{V_{w d}(t)} d t
$$

Below, we describe how $\rho_{b a l l}$ and $V_{w d}$ may vary with time.

Even before impact, incoming comets may split as they are heated and/or tidally disrupted. However, the resulting fragmentation speeds of $\sim 0.1 \mathrm{~km} \mathrm{~s}^{-1}$ (Sekanina 1982) are vastly smaller than the impact speeds that exceed $1000 \mathrm{~km} \mathrm{~s}^{-1}$. We therefore neglect the small deviations from the initial hyperbolic orbits that arise. In any case, the loss of some fragments that deviate away from a path that leads them into a direct collision is balanced by the gain of other fragments from parent bodies on a near-miss trajectory that deviate into a collision-course.

For simplicity, we assume $V_{w d}$ is dominated by the white dwarf's space motion in the Local Standard of Rest and we ignore the motions of the comets. Assuming that only the motion vertical to the plane varies significantly with time, we write:

$$
V_{w d}=\left(U^{2}+V^{2}+W(t)^{2}\right)^{1 / 2}
$$

with $U, V$ and $W$ having their conventional meanings. We adopt the simplifying assumption that relative to the Galactic Plane a star undergoes vertical simple harmonic motion (Kuijken \& Gilmore 1989)

$$
W(t)=W_{0} \cos k^{1 / 2} t
$$

With $k^{1 / 2}=2.3 \times 10^{-15} \mathrm{~s}^{-1}$ (Siebert et al. 2003), the period of a star's oscillation through the Galactic Plane is 86 Myr.

We assume that interstellar iceballs have the same spatial distribution as F-type stars and therefore relative to the Galactic Plane:

$$
\rho_{\text {ball }}(H)[z]=\rho_{\text {ball }}(H)[0] e^{-|z| / h}
$$


We adopt $h=150$ pc (Gilmore \& Zeilik 2000).

Our analysis only applies to iceballs in a limited size range. Small iceballs are significantly sublimated on their approach to the white dwarf, and the resulting gas atoms may not accrete onto the star. Reformulating slightly Equation (14) in Jura (2005), the thickness, $\Delta R$, of a comet which is thermally sublimated as it approaches a star is:

$$
\Delta R \approx \frac{L_{*} m_{H_{2} O} \Delta \phi_{s u b l}}{16 \pi \Delta E\left[2 G R_{*} M_{*}\right]^{1 / 2}}
$$

where $m_{H_{2} \mathrm{O}}$ is the mass of a water molecule, $\Delta \phi_{\text {subl }}$ is the angular variation in its orbit traced by the incoming comet as seen by the host star where ice sublimation dominates the cooling of the white dwarf, $\Delta E$ is the energy per molecule associated with sublimation (taken equal to $2 \times 10^{-12}$ erg, Sekanina (2002)) and we assume an orbit that is nearly parabolic. We take $\Delta \phi_{\text {subl }}=\pi / 2$ since ice sublimates significantly at distances much greater than $R_{*}$. For illustrative purposes, we take $L_{*}=0.05 \mathrm{~L}_{\odot}, R_{*}=0.013 \mathrm{R}_{\odot}$ and $M_{*}=0.6 \mathrm{M}_{\odot}$ as appropriate for the white dwarfs of interest. We find that $\Delta R \approx 240 \mathrm{~cm}$. Therefore, iceballs with radii greater than $\sim 10 \mathrm{~m}$ deposit most of their mass onto the white dwarf. Our analysis only constrains iceballs with hydrogen masses less than the upper limits of accreted hydrogen in our target white dwarfs. This implies that we consider iceballs with less than $4 \times 10^{15} \mathrm{~g}$ of hydrogen and therefore a total mass less than $8 \times 10^{16} \mathrm{~g}$ and a radius less than $2 \mathrm{~km}$.

Besides thermal sublimation, is there some other process which leads to a comet's disintegration and would the resulting gas and debris somehow be inhibited from accreting onto the star? In considering this possibility, it should be recognized that the approach of a comet to a white dwarf is less disruptive than the approach of a comet to the Sun for two reasons. First, the white dwarf's luminosity is lower than the Sun's and thus a comet nearing a white dwarf is heated less and the resulting thermal stresses are smaller. Second, the typical white dwarf mass of $0.6 \mathrm{M}_{\odot}$ is less than the Sun's mass and therefore the gravitational tidal forces from a white dwarf are smaller. As a result, while comets may split as they approach their target white dwarf, analogous to the approach of comets to the Sun, we do not expect them to fully disintegrate - if they ever do - until they are closer than $\sim 1 R_{\odot}$. At this separation, the comet's incoming speed is nearly $500 \mathrm{~km} \mathrm{~s}^{-1}$ and it survives less than $1000 \mathrm{~s}$ before impact. Perhaps as the comet nears within $1 \mathrm{R}_{\odot}$ of the white dwarf, there may be extensive explosions. Generalizing from the observations of comet $17 \mathrm{P} /$ Holmes which displayed remarkable transient brightening (Reach et al. 2010), the typical outflow speed of dust fragments and gas debris in such events might be $0.1 \mathrm{~km} \mathrm{~s}^{-1}$. At this speed, the ejected material travels $\sim 100 \mathrm{~km}$ in the comet's frame of reference before impacting the white dwarf. Since this transverse distance is much smaller than the radius of the white dwarf of nearly $10^{4}$ $\mathrm{km}$, we expect that cometary material that initially is on a trajectory aimed at the star does in fact impact, regardless of any explosions that occur during the last $1000 \mathrm{~s}$. This argument applies even to small dust grains. The luminosity of a white dwarf is so low that even tiny solid particles cannot be substantially deflected by radiation pressure (see Section 5.6 of Plavchan et al. (2005)). In conclusion, even if a comet largely disintegrates during its final approach to a white dwarf, the 
bulk of the material is accreted.

\subsection{Application to GD 190 and BPM 17088}

To date, the most sensitive upper limits to hydrogen in DBs are by Petitclerc et al. (2005) who report ultraviolet spectroscopy for three stars. One of the stars, EC 20058-5234 with $T_{*}$ $=28,000 \mathrm{~K}$, is relatively young and just beginning to have a well developed convective zone (Gautschy \& Althaus 2002). Our toy model described in Section 2 may not apply. Therefore, we only quantitatively discuss results for GD 190 and BPM 17088. The parameters that we adopt for the two targets and relevant references are given in Table 1. If their effective temperatures are less than 22,000 K, then our derived upper limits to the space density of interstellar comets are weakened.

From Equation (8), the maximum distance that a star travels from the Galactic Plane is $W_{0} / k^{1 / 2}$. If $W_{0}$ equals the value of $W$ given in Table 1 , then GD 190 and BPM 17088 remain within $150 \mathrm{pc}$, the estimated scale height around the Galactic Plane of the interstellar iceballs. Using the parameters in Table 1, and making the simplifying assumption that the interstellar density and speed of the white dwarf are constant since $|W(t)|<<V_{w d}$ and $|z|<h$, then from Equation (6), the upper limits to $\rho_{\text {ball }}(H)$ experienced by GD 190 and BPM 17088 are $1.5 \times 10^{-29} \mathrm{~g}$ $\mathrm{cm}^{-3}$ and $1.0 \times 10^{-29} \mathrm{~g} \mathrm{~cm}^{-3}$, respectively. We assume that interstellar comets are mainly ice and silicates and that $\rho(O) \approx 10 \rho(H)$ (Greenberg 1998). Consequently the upper bounds to $\rho_{\text {ball }}(O)$ in the Galactic midplane are $1.5 \times 10^{-28} \mathrm{~g} \mathrm{~cm}^{-3}$ and $1.0 \times 10^{-28} \mathrm{~g} \mathrm{~cm}^{-3}$ for GD 190 and BPM 17088, respectively. Therefore, we find that the density of oxgyen contained within large iceballs is less than about $1 \%$ of the total interstellar oxygen density, and that "optimistic" models for the space density of interstellar comets (Section 1.2) with radii between $10 \mathrm{~m}$ and $2 \mathrm{~km}$ are excluded.

Above, we assumed that any wind from the white dwarf is completely negligible. We now consider the implications of including a wind and show in Figure 3 results for the amount of hydrogen accumulated by a DB as a function of time for a case where the average accretion rate is $10 \mathrm{~g} \mathrm{~s}^{-1}$, a factor of four above the values given in Table 1 . We see that if $\dot{M}_{\text {wind }}$ is less than $10^{6} \mathrm{~s}^{-1}$, more hydrogen is accumulated than allowed by the observed upper bound of $4 \times 10^{15} \mathrm{~g}$. Therefore, if warm white dwarf winds are plausibly weak, interstellar iceballs are rare.

\section{OBSERVATIONAL POSSIBILITIES}

We have suggested that DBs warmer than 22,000 K are not polluted by ice-rich asteroids, comets or interstellar gas. This scenario can be tested by examining atmospheric compositions. Using ground-based observations, Zuckerman et al. (2010) found that over 30\% of DBs between $13,500 \mathrm{~K}$ and $19,500 \mathrm{~K}$ display heavy atoms. To date, the most extensive similar study of DBs with $T_{*}>22,000 \mathrm{~K}$ is by Voss et al. (2007). In this sample of 11 stars, only WD $2354+1659$ was 
identified as having heavy atoms in its photosphere. One possibility is that this star has accreted icepoor asteroids as could be inferred with a detailed abundance analysis similar to that performed by Klein et al. (2010) for GD 40. Also, however, the temperature of this star is uncertain. Depending upon the gravity and hydrogen content, $T_{*}$ could be as high as 24,800 K (Voss et al. 2007) or as low as 19,000 K (Koester et al. 2005). While the frequency of externally-polluted "warm" DBs appears to be low compared to the cooler DBs, further measurements should be performed since the spectra obtained by Voss et al. (2007) are not as sensitive to heavy atoms as those obtained by Zuckerman et al. (2010).

Ultraviolet spectroscopy is a sensitive tool to measure heavy elements in a white dwarf's atmosphere (Desharnais et al. 2008). Data from the Far Ultraviolet Spectroscopic Explorer and the Hubble Space Telescope have been obtained for 5 DBs warmer than 22,000 K: GD 190, BPM 17088, EC 20058-5234 (Petitclerc et al. 2005), PG 0112+104 (Provencal et al. 2000) and GD 358 (Provencal et al. 1996). Although these stars possess atmospheric carbon, it is almost certain that this heavy element is intrinsic to the star and not accreted (Fontaine \& Brassard 2005, Desharnais et al. 2008). With the models of Koester (2009), the upper limits to the iron abundance found by Petitclerc et al. (2005) and the assumption that $1 / 3$ of the accreted mass is iron as seems appropriate for extrasolar asteroids (Klein et al. 2010; Zuckerman et al. 2007, 2010; Dufour et al. 2010; Vennes et al. 2010), then in GD 190, for example, the upper limit to the heavy atom accretion rate is $\sim 1 \times 10^{6} \mathrm{~g} \mathrm{~s}^{-1}$, a value lower than any reported by Zuckerman et al. (2010) for polluted cooler DBs. Therefore ultraviolet results are more sensitive than optical studies for identifying pollution. If each ultraviolet targeted DB has at least a $30 \%$ probability of experiencing pollution, as found by Zuckerman et al. (2010) for cool DBs, then the total probability that all 5 atars are uncontaminated is less than $(0.7)^{5}$ or $16 \%$. Future observations of warm DBs may further test the prediction that these stars do not accrete ice-rich asteroids.

The warmest DA known to have a dust disk is SDSS $1228+1040$ with $T_{*}=22,600 \mathrm{~K}$ (Gaensicke et al. 2006; Brinkworth et al. 2009; Melis et al. 2010). Discovery of additional warm DAs with dust and then measuring their pollution may help extend the argument described in Section 2 to constrain the mass loss rates from such stars.

In our toy model described in Section 2, we have treated hydrogen accretion as a minor perturbation in a DB atmosphere. In fact, it may take only $\sim 10^{18} \mathrm{~g}$ of hydrogen to prevent the conversion of a DA into a DB for stars warmer than 22,000 K by dredge-up of interior helium (MacDonald \& Vennes 1991; Su \& Li 2010). In contrast, for cooler stars with much more massive helium convective zones, considerably more hydrogen may be required to prevent the conversion from DAs to DBs (MacDonald \& Vennes 1991; Tremblay et al. 2008). The fraction of all white dwarfs that are DBs with $T_{*}$ between $25,000 \mathrm{~K}$ and $40,000 \mathrm{~K}$ is a factor of 2.5 less than the same fraction of DBs with $T_{*}$ between 16,000 K and 22,000 K (Eisenstein et al. 2006). We speculate that the systems with ice-rich asteroids accrete enough hydrogen to prevent a white dwarf from becoming a DB until the temperature falls appreciably below 22,000 K. 
Our supposition that the Oort cloud is unusually massive is consistent with the previous suggestion by Shull \& Stern (1995), and the picture that the solar system is somewhat special. The orbital eccentricities of our planets are unusually low (Marcv \& Butler 2000) and extrasolar analogs to the Late Heavy Bombardment appear to be rare (Booth et al. 2009).

\section{CONCLUSIONS}

We identify a subclass of warm DBs where accretion of asteroids, analogs to Oort clouds comets and interstellar gas all appear to be negligible. We make the plausible argument that the wind mass loss rate these stars is less than $10^{6} \mathrm{~g} \mathrm{~s}^{-1}$. If these stars have their nominal effective temperatures, it then follows that because they have accumulated only a small mass of hydrogen, interstellar comets with radii between $10 \mathrm{~m}$ and $2 \mathrm{~km}$ a contain less than $1 \%$ of all interstellar oxygen. "Optimistic" scenarios for the formation of the solar system's Oort cloud may not apply to most other stars.

This work has been partly supported by the National Science Foundation. I thank B. Klein, M. Kilic, D. Koester, S. Xu and B. Zuckerman for helpful comments.

\section{REFERENCES}

Alcock, C., Fristrom, C. C., \& Weigelman, R. 1986, ApJ, 302, 462

Asplund, M., Grevesse, N., Sauval, A. J., Allende Prieto, C., \& Kiselman, D. 2004, A\&A, 417, 751

Beauchamp, A., Wesemael, F., Bergeron, P., Fontaine, G., Saffer, R. A., Liebert, J., \& Brassard, P. 1999, ApJ, 516, 887

Bohlin, R. C., Savage, B. D., \& Drake, J. F. 1978, ApJ, 224, 132

Booth, M., Wyatt, M. C., Morbidelli, A., Moro-Martin, A., \& Levison, H. F. 2009, MNRAS, 399, 385

Brinkworth, C. S., Gaensicke, B. T., Marsh, T. R., Hoard, D. W., \& Tappert, C. 2009, 696, 1402

Caffau, E., Ludwig, H.-G., Steffen, M., Ayres, T. R., Bonifacio, P., Cayrel, R., Freytag, B., \& Plez, B. $2008, A \& A, 488,1031$

Cartledge, S. I. B., Lauroesch, J. T., Meyer, D. M., \& Sofia, U. J. 2004, ApJ, 613, 1037

Delahaye, F., \& Pinsonneault, M. H. 2006, ApJ, 649, 529

Desharnais, S., Wesemael, F., Chayer, P., Kruk, J. W., \& Saffer, R. A. 2008, ApJ, 672, 540

Dones, L., Weissman, P. R., Levison,H. F., \& Duncan, M. J. 2004, in Comets II, ed. M. C. Festou, U. Keller, \& H. A. Weaver (Tucson, AZ: Univ. of Arizona), 153 
Dufour, P., et al. 2007, ApJ, 663, 1291

Dufour, P., Kilic, M., Fontaine, G., Bergeron, P., Lachapelle, F.-R., Kleinman, S. J., \& Leggett, S. K. 2010, ApJ, 719, 803

Duncan, M., Quinn, T., \& Tremaine, S. 1987, AJ, 94, 1330

Dupuis, J., Fontaine, G., Pelletier, C., \& Wesemael, F. 1992, ApJS, 82, 505

Eisenstein, D. J. et al. 2006, AJ, 132, 676

Farihi, J., Barstow, M., Redfield, S., Dufour, P., \& Hambly, N. C. 2010, MNRAS, 404, 2123

Farihi, J., Jura, M., \& Zuckerman, B. 2009, ApJ, 694, 805

Fontaine, G., \& Brassard, P. 2005, in ASP Conf. Ser. 334, 14th European Workshop on White Dwarfs, ed. D. Koester \& S. Moehler (San Francisco, CA: ASP), 49

Francis, P. J. 2005, ApJ, 635, 1348

Gaensicke, B. T., Marsh, T. R., \& Southworth, J. 2007, MNRAS, 380, L35

Gaensicke, B. T., Marsh, T. R., Southworth, J., Rebassa-Mansergas, A. 2006, Science, 314, 1908

Gautschy, A., \& Althaus, L. G. 2002, A\&A, 382, 141

Gilmore, G., \& Zeilik, M. 2000, in Allen's Astrophysical Quantities, ed. A. N.Cox (New York: Springer), 471

Greenberg, J. M. 1974, ApJ, 189, L81

Greenberg, J. M. 1998, A\&A, 330, 375

Hansen, B. M. S., \& Liebert, J. 2003, ARA\&A, 41, 465

Jenkins, E. B. 2009, ApJ, 700, 1299

Jura, M. 2003, ApJ, 584, L91

Jura, M. 2005, AJ, 130, 1261

Jura, M., Muno, M. P., Farihi, J., \& Zuckerman, B. 2009, ApJ, 699, 1473

Jura, M. \& Xu, S. 2010, AJ, 140, 1129

Kilic, M., von Hippel, T., Leggett, S. K., \& Winget, D. E. 2006, ApJ, 646, 474

Klein, B., Jura, M., Koester, D., Zuckerman, B., \& Melis, C. 2010, ApJ, 709, 950

Koester, D. 2009, A\&A, 498, 517 
Koester, D., Rollenhagen, K., Napiwotzki, R., Voss, B., Christlieb, N., Homeier, D., \& Reimers, D. 2005, A\&A, 432, 1025

Kuijken, K., \& Gilmore, G. 1989, MNRAS, 239, 571

Lamers, H. H. G. L. M., \& Cassinelli, J. 1999, Introduction to Stellar Winds (Cambridge: Cambridge University Press)

Levison, H., Duncan, M. J., Brasser, R., \& Kaufmann, D. E. 2010, Science, 329, 187

Liebert, J., Wesemael, F., Hansen, C. J., Fontaine, G., Shipman, H L., Sion, E. M., Winget, D. E., \& Green, R. F. 1986, ApJ, 309, 241

MacDonald, J., \& Vennes, S. 1991, ApJ, 371, 719

Marcy, G. W.,\& Butler, R. P. 2000, PASP, 112, 137

McGlynn, T. A., \& Chapman, R. D. 1989, ApJ, 346, L105

Melis, C., Jura, M., Albert, L., Klein, B., \& Zuckerman, B. 2010, ApJ, 722, 1078

Musielak, Z. E., Noble, M., Porter, J. G., \& Winget, D. E. 2003, ApJ, 593, 481

O’Dwyer, I. J., Chu, Y.-H., Gruendl, R. A., Guerrero, M. A., \& Webbink, R. F. 2003, AJ, 125, 2239

Parriott, J., \& Alcock, C. 1998, ApJ, 501, 357

Petitclerc, N., Wesemael, F., Kruk, J. W., Chayer, P., \& Billeres, M. 2005, ApJ, 624, 317

Plavchan, P., Jura, M., \& Lipscy, S. 2005, ApJ, 631, 1161

Provencal, J. L., Shipman, H. L., Thejll, P., Vennes, S., \& Bradley, P. A. 1996, ApJ, 466, 1011

Provencal, J. L., Shipman, H. L., Thejll, P., \& Vennes, S. 2000, ApJ, 542, 1041

Provencal, J. L. et al. 2009, ApJ, 693, 564

Reach, W. T., Vaubaillon, J., Lisse, C. M., Holloway, M., \& Rho, J. 2010, Icarus, 208, 276

Sekanina, Z. 1976, Icarus, 27, 123

Sekanina, Z. 1982, in Comets, ed. L. Wilkening (Tucson: University of Arizona), 251

Sekanina, Z. 2002, ApJ, 566, 577

Sen, A. K., \& Rama, N. C. 1993, A\&A, 275, 298

Shull, J. M., \& Stern, S. A. 1995, AJ, 109, 690 
Siebert, A., Bienayme, O., \& Soubiran, C. 2003, A\&A, 399, 531

Sion, E. M., Fritz, M. L., McMullin, J. P., \& Lallo, M. D. 1988, AJ, 96, 251

Spitzer, L. 1978, Physical Processes in the Interstellar Medium (New York: J. Wiley)

Stern, S. A. 1990, PASP, 102, 793

Stern, S. A., \& Shull, J. M. 1990, ApJ, 359, 506

Su, J., \& Li, Y. 2010, Res. Astron. Astrophys. 10, 266

Sullivan, D. J. et al. 2008, MNRAS, 387, 137

Tremblay, P.-E., \& Bergeron, P. 2008, ApJ, 672, 1144

Unglaub, K. 2008, A\&A, 486, 923

Unglaub, K., \& Bues, I. 2000, A\&A, 359, 1042

Vennes, S., Kawka, A., \& Nemeth, P. 2010, MNRAS, 404, L40

von Hippel, T., Kuchner, M. J., Kilic, M., Mullally, F., \& Reach, W. T. 2007, ApJ, 662, 544

Voss, B., Koester, D., Napiwotzki, R., Christlieb, N., \& Reimers, D. 2007, A\&A, 470, 1079

Weissman, P. R. 1996, in ASP Conf. Ser. 107, Completing the Inventory of the Solar System, ed. T. W. Rettig \& J. M. Hahn (San Francisco: ASP) 265

Whipple, F. L. 1975, AJ, 80, 525

Whittet, D. C. B. 2010, ApJ, 710, 1009

Zuckerman, B., Koester, D., Melis, C., Hansen, B., \& Jura, M. 2007, ApJ, 671, 872

Zuckerman, B., Melis, C., Klein, B., Koester, D., \& Jura, M. 2010, ApJ, 722, 725 
Table 1 - Target White Dwarfs

\begin{tabular}{|c|c|c|c|}
\hline & GD 190 & BPM 17088 & Reference(s) \\
\hline$T_{*}(\mathrm{~K})$ & 22,000 & 22,600 & $a$ \\
\hline $\log g(\operatorname{cgs})$ & 7.97 & 8.07 & $a, b$ \\
\hline$M_{*}\left(\mathrm{M}_{\odot}\right)$ & 0.59 & 0.64 & $a, b$ \\
\hline $\log n(\mathrm{H}) / n(\mathrm{He})$ & $\leq-5.5$ & $\leq-5.5$ & $a$ \\
\hline $\log M_{C V Z}(\mathrm{~g})$ & 21.73 & 21.61 & $c$ \\
\hline $\log M_{a c c}(H)(\mathrm{g})$ & $\leq 15.63$ & $\leq 15.51$ & \\
\hline $\log t_{c o o l}(\mathrm{Myr})$ & 7.70 & 7.64 & $d$ \\
\hline $\log R_{*}(\mathrm{~cm})$ & 8.96 & 8.93 & \\
\hline$\dot{M}_{a c c}(H)\left(\mathrm{g} \mathrm{s}^{-1}\right)$ & $\leq 2.7$ & $\leq 2.4$ & \\
\hline$U\left(\mathrm{~km} \mathrm{~s}^{-1}\right)$ & -19 & 14 & $e$ \\
\hline$V\left(\mathrm{~km} \mathrm{~s}^{-1}\right)$ & -12 & -10 & $e$ \\
\hline$W\left(\mathrm{~km} \mathrm{~s}^{-1}\right)$ & -9 & 8 & $e$ \\
\hline$V_{w d}\left(\mathrm{~km} \mathrm{~s}^{-1}\right)$ & 24 & 19 & \\
\hline
\end{tabular}

${ }^{a}$ Petitclerc et al. (2005), ${ }^{b}$ Voss et al. (2007), ${ }^{c}$ Equation (4) and ignoring the mass variation of this quantity, ${ }^{d}$ Equation (5) and ignoring the mass variation in this quantity, ${ }^{e}$ Sion et al. (1988) 


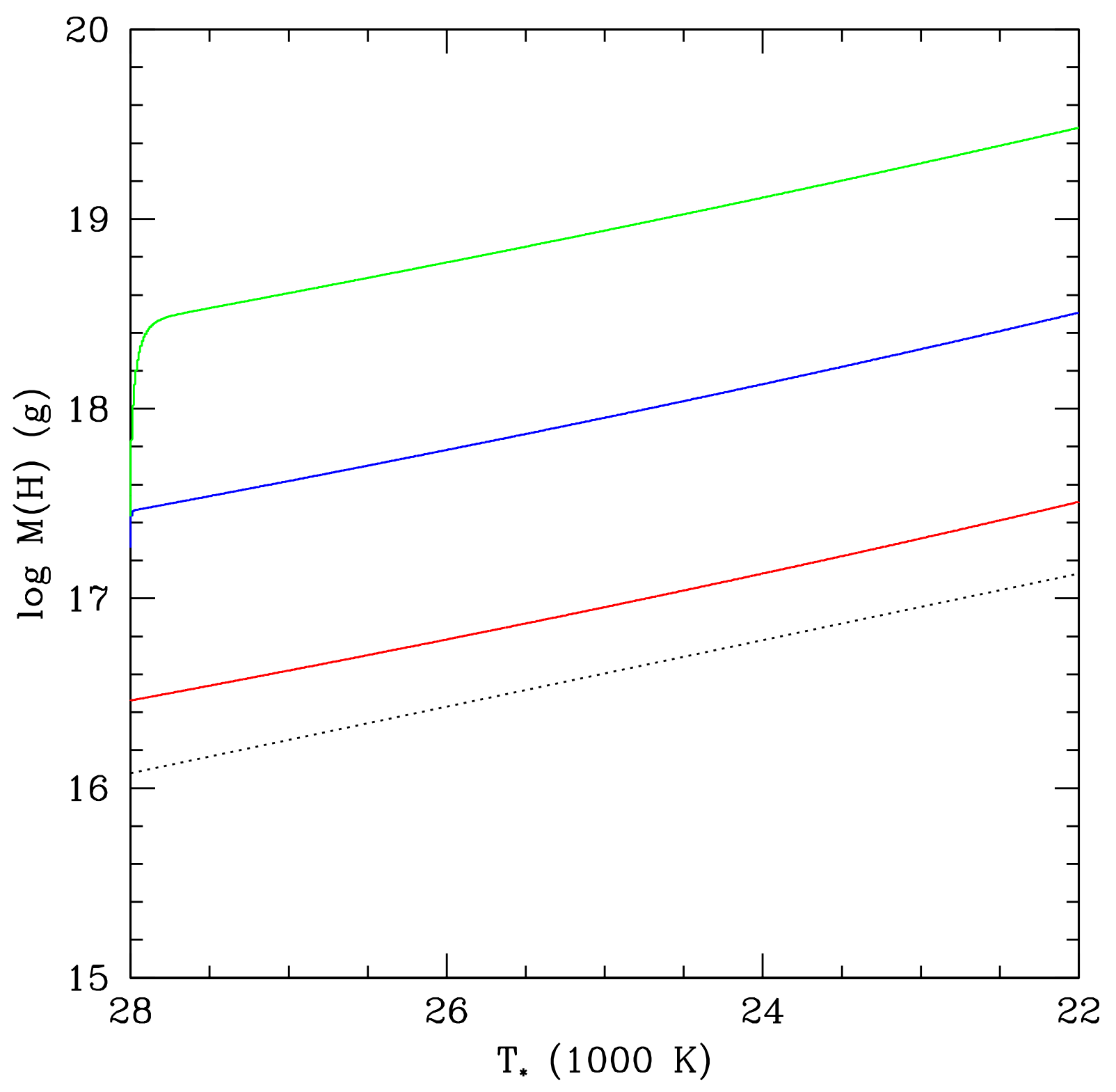

Fig. 1.- Predicted accumulated hydrogen mass from asteroidal accretion as a function of effective temperature for different wind mass loss rates. We use $M(H)[0]=1.0 \times 10^{15} \mathrm{~g}, \dot{M}_{a c c}(H)=6 \times$ $10^{5} \mathrm{~g} \mathrm{~s}^{-1}$ and values of $\dot{M}_{\text {wind }}$ of $10^{10}, 10^{9}$ and $10^{8} \mathrm{~g} \mathrm{~s}^{-1}$ shown by the red, blue and green lines, respectively. The dotted line represents the upper limit to hydrogen in a DB with the assumptions that $n(\mathrm{H}) / n(\mathrm{He}) \leq 10^{-4}$ and $M_{c v z}$ given by Equation (4). We see that in the likely case that $\dot{M}_{w i n d}$ $<10^{10} \mathrm{~g} \mathrm{~s}^{-1}$, the assumption that the star accretes ice-rich asteroids over-predicts the amount of accumulated hydrogen 


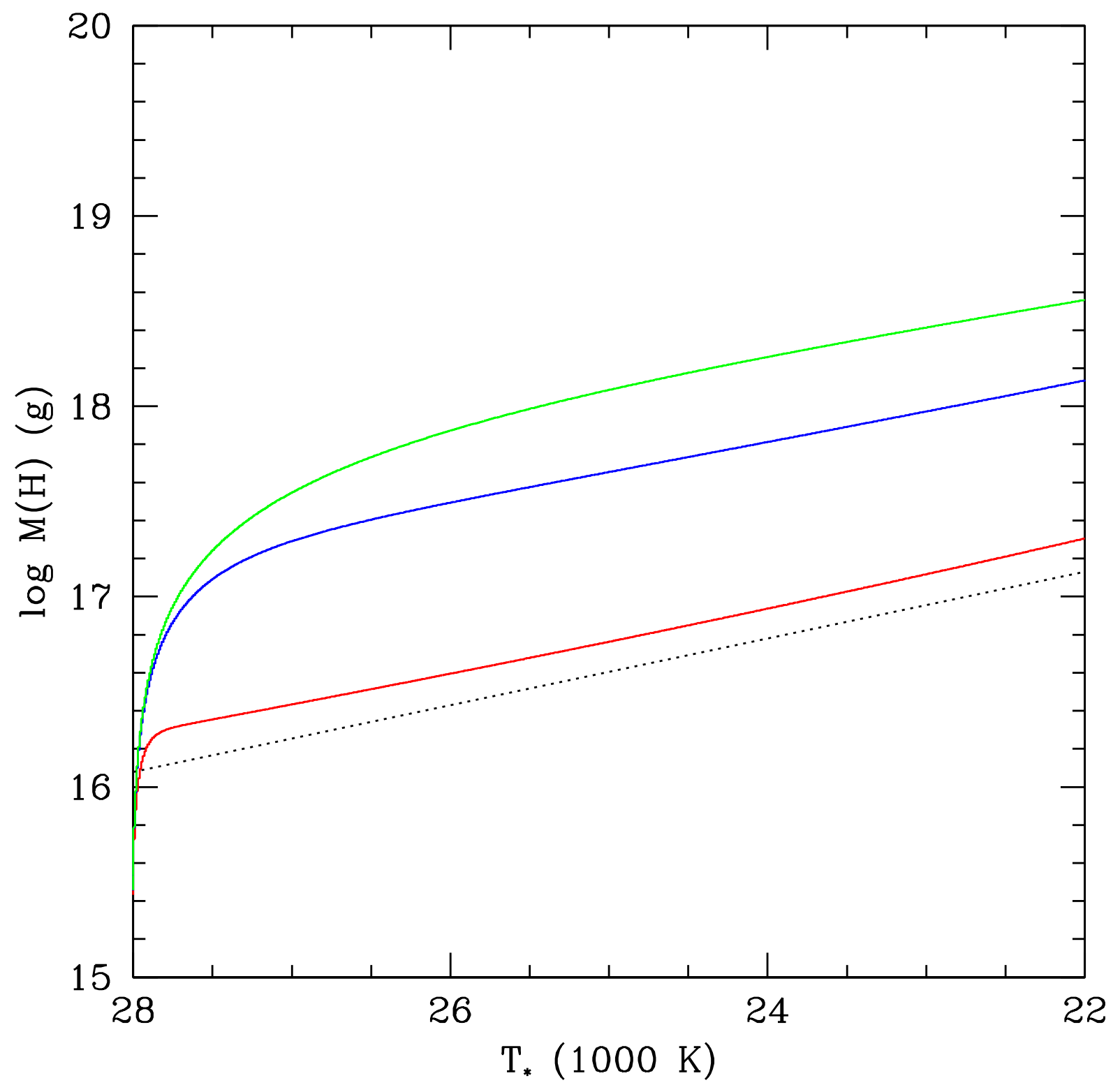

Fig. 2. - Similar to Figure 1 except for accretion from an analog to the Oort cloud with $\dot{M}_{a c c}(H)$ $=4 \times 10^{3} \mathrm{~g} \mathrm{~s}^{-1}$ and values of $\dot{M}_{\text {wind }}$ of $10^{8}, 10^{7}$ and $10^{6} \mathrm{~g} \mathrm{~s}^{-1}$ shown by the red, blue and green curves, respectively. We see that in the plausible case that $\dot{M}_{\text {wind }}<10^{8} \mathrm{~g} \mathrm{~s}^{-1}$, the assumption that the gas accretes comets from a conservative extrapolation of our own Oort cloud over-predicts the amount of accumulated hydrogen. 


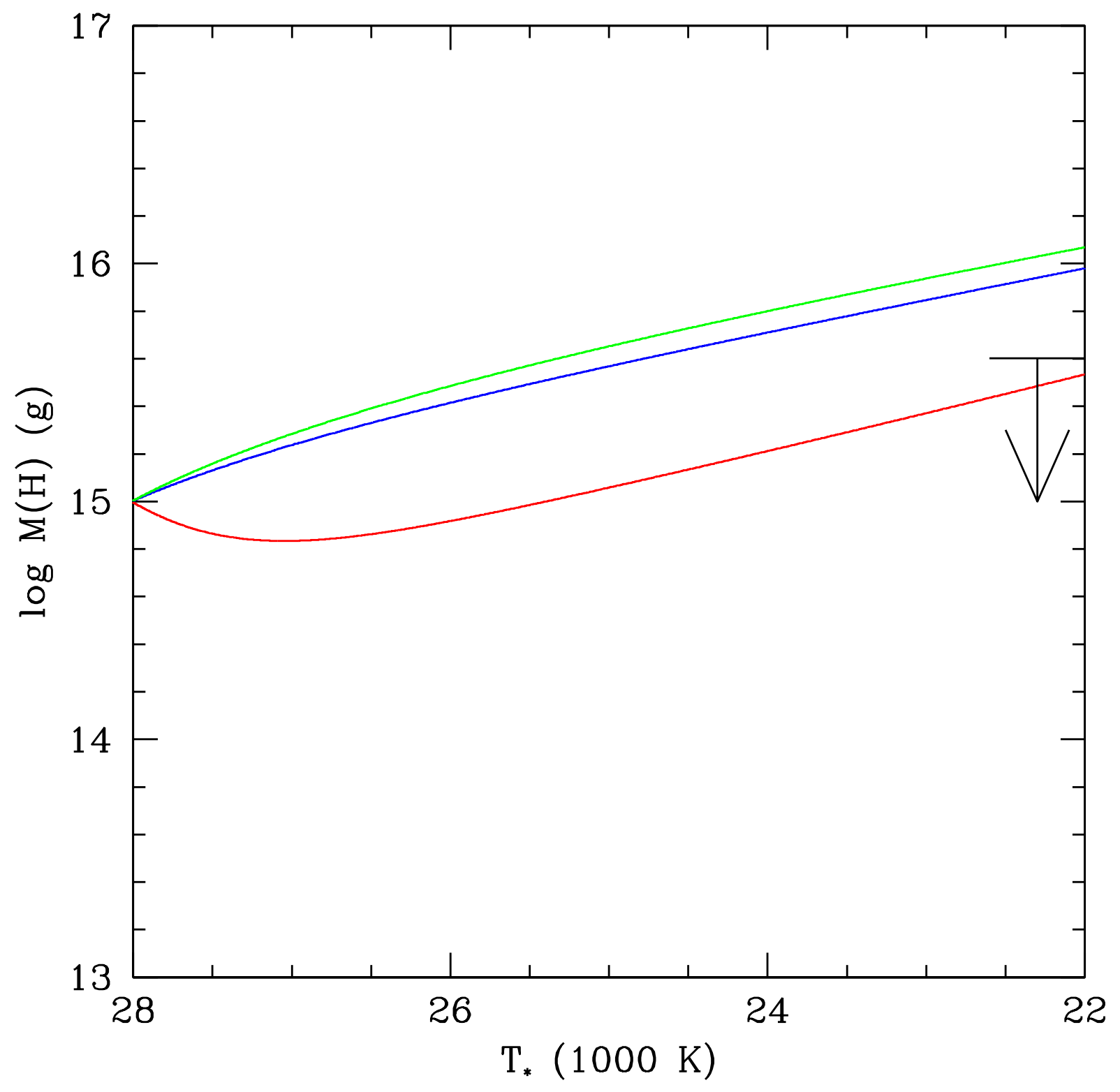

Fig. 3.- Similar to Figure 1 except for an accretion rate of $\dot{M}_{a c c}=10 \mathrm{~g} \mathrm{~s}^{-1}$ and values of $\dot{M}_{\text {wind }}$ of $10^{7}, 10^{6}$ and $10^{5} \mathrm{~g} \mathrm{~s}^{-1}$ shown by the red, blue and green curves, respectively. The downward pointing arrow represents the upper bound to the mass of accumulated hydrogen in GD 190 at the temperature range of the two DBs in Table 1 . We see that in the plausible case that $\dot{M}_{\text {wind }}<10^{6}$ $\mathrm{g} \mathrm{s}^{-1}$, more hydrogen is predicted to be accumulated than observed. 